\title{
6 \\ Using the program applications on the BBC Model B microcomputer
}

The program applications outlined in the text may be used on the UltraCalc spreadsheet format developed by BBC Software for the BBC Model B computer.

The UltraCalc software ROM chip may be purchased from any BBC dealership and is easily fitted into the computer. Full fitting instructions are included in the UltraCalc Manual.

The following program listings may be directly input on to the UltraCalc spreadsheet format.

\subsection{Project Cash Flow}

2.2 Company Cash Flow

2.4 Cash Flow - Labour, Plant, Material and Subcontractor Analysis

3.1 Monthly Ledger Account

3.2 Present Value Assessment

3.4 Monthly Cost/Value Report

4.1 Subcontractor's Payment

4.2 Valuation Statement

4.3 Budget Estimate (Brickwork)

4.4 Development Budget

4.5 Present Value/Annual Equivalent

4.6 Painting Estimate

5.1 Reduced Levelling Applications

5.4 Offsets from the Main Chord.

The user must acquaint himself with the UltraCalc routines and commands prior to inputting the program listings.

The following program listings from chapter 5 include the use of the integer command, INT $(x)$, of the VisiCalc functions.

\subsection{Closed Traverse Calculation}

5.3 Circular Curve - Deflection Angles

5.5 Stadia Tacheometry

5.6 Open Traverse Reduction.

In order to assist the user, the following differences between the VisiCalc and UItraCalc routines and com- mands are given. They will be of interest when inputting program listings or formatting the screen layout.

The integer command on VisiCalc, INT(x), may be substituted on UItraCalc by FIX(x)

The @ sign which prefixes all functions on the VisiCalc listings is not required on UltraCalc.

As there is no LABEL REPEATING routine (/-) on UltraCalc, the user can simply format the screen layout using the REPLICATE routine to establish a row of dashes. Alternatively, and much more simply, the dash key can be held down to repeat a row of dashes across the cell locations to form a line.

The standard UltraCalc column width is seven characters. This must be adjusted by the user to suit the layout display on the screen. Care must be taken when entering titles that on VisiCalc run from one column to another. The user may adjust the UltraCalc column width to suit the titles or set up his own titles or headings on the screen, using the screen displays illustrated as a guide to the layout.

When entering numbers on the reduced level display (example 5.1), or any values to decimal places, the following routine must be followed on UltraCalc. Input the number (1.375) and press return $@$. The number will appear in the cell location to the nearest whole number (1.00). Now

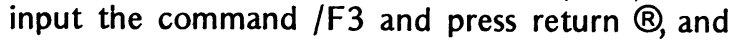
the number in the cell location will change to 1.375 .

An applications disk has been produced for the BBC Model B microcomputer, containing a selection of the applications discussed in the text. Refer to appendix III for details on how to obtain the disk. 